\title{
REVISTA ECONOMÍA
}

\section{Análisis de la pobreza multidimensional en los hogares de la agricultura familiar campesina en el Ecuador, 2009-2019}

\author{
Melany Morales | iD Pontificia Universidad Católica del Ecuador (Ecuador) \\ Andrés Mideros | iD Pontificia Universidad Católica del Ecuador (Ecuador)
}

\begin{abstract}
RESUMEN El artículo analiza la pobreza multidimensional en los hogares de la agricultura familiar y campesina ( $\mathrm{AFC}$ ) entre 2009 y 2019, identificando sus principales carencias y comparándolos con hogares a nivel nacional y rural. Se evidencia el aumento de la brecha de empobrecimiento de la AFC a lo largo del periodo. Más de la mitad de los hogares de la AFC presentan privaciones conjuntas en las cuatro dimensiones: educación; trabajo y seguridad social; salud, agua y alimentación; y hábitat, vivienda y ambiente sano. Las principales carencias de la AFC se evidencian en falta de acceso al agua, desempleo o empleo inadecuado, logro educativo incompleto, no contribución al sistema de pensiones y déficit habitacional.
\end{abstract}

PALABRAS CLAVE Agricultura familiar y campesina, pobreza multidimensional, desigualdad.

FECHA DE RECEPCIÓN 29/09/2021 FECHA DE REVISIÓN 01/11/2021 FECHA DE APROBACIÓN 04/11/2021

Multidimensional poverty in peasant families farming in Ecuador, 2009-2019

ABSTRACT The article studies multidimensional poverty in peasant families farming (PFF) in the period 2009 and 2019 and compares it with households at national and rural levels. The raise of the impoverishment gap is evident throughout the period against PFF. More than half of the households in the PFF show deprivation in all four dimensions together: education; labor and social security; health, water, and food; and housing and environment. The main deprivations are access to water, unemployment or inadequate employment, incomplete educational achievement, non-contribution to the pension system and housing deficit.

KEY WORDS Peasant family farming, multidimensional poverty, inequality.

JEL CODES I30, D1, Q19.

\section{INTRODUCCIÓN}

La agricultura familiar campesina (AFC) concentra al 11\% de la población del Ecuador, representa cerca del $75 \%$ del total de unidades productivas agropecuarias (UPA) y ocupa el 17\% de la superficie de uso agrícola del Ecuador (Instituto Interamericano de Cooperación para la Agricultura [IICA], s. f.). La AFC incluye al conjunto de actividades agrícolas, forestales, acuícolas, pesqueras y pecuarias, realizadas por un núcleo familiar, es decir, que depende de la mano de obra de 
las personas que conforman el hogar (Organización de las Naciones Unidas para la Alimentación y la Agricultura [FAO], 2014). En la AFC existe un equilibrio entre la familia y el emprendimiento porque poseen el control de los recursos - tierra, maquinaria, material genético y experiencia- y la ganancia o la obtención de utilidades por las actividades realizadas se vuelve a invertir en la unidad productiva o para la reproducción familiar (Van Der Ploeg, 2014).

Sin embargo, es un sector afectado por limitaciones de acceso a factores productivos, recursos económicos y mercados, así como por falta de infraestructuras y tecnologías adecuadas, desigualdades de género y carencia de renovación generacional (FAO, 2018a), restricciones de acceso a servicios financieros y servicios básicos (Van Der Ploeg, 2014). Adicionalmente, se encuentra condicionada por factores histórico-institucionales como la concentración de la propiedad de la tierra (Chonchol, 2008).

La AFC es un actor clave para el desarrollo rural, reducir la pobreza, erradicar el hambre, e incluso para la conservación tanto de tradiciones culturales como de biodiversidad y lograr un cambio hacia sistemas agrícolas sostenibles (Van Der Ploeg, 2014). Al mismo tiempo, tiene un papel esencial para alcanzar la soberanía alimentaria, ya que un gran porcentaje de producción agrícola está a cargo de este sistema de producción, así como también la mayoría de los alimentos que se consumen. En el caso del Ecuador, el 64\% de la producción agrícola y 60\% de los alimentos consumidos en el país provienen de la AFC (FAO, s. f.).

Cabe señalar que la agricultura presenta un detrimento en la participación relativa en comparación con los demás sectores, muestra baja productividad y alta proporción de población rural, e incluso exhibe brechas de productividad interna entre los agentes o actividades del mismo sector; es decir, las economías campesinas frente a la agroindustria (Comisión Económica para América Latina y Organización de las Naciones Unidas [CEPAL/ONU], 2010). En cuanto a la estructura interna de la AFC, la carencia de renovación generacional es uno de los retos. Los jóvenes migran hacia las ciudades y el extranjero, porque se percibe a la agricultura como una actividad con baja remuneración (Comisión Económica para América Latina, Organización de las Naciones Unidas para la Alimentación y la Agricultura e Instituto Interamericano de Cooperación para la Agricultura [CEPAL/FAO/IICA], 2014).

La globalización y la participación de las corporaciones transnacionales imponen patrones de consumismo, ocasionando altos costos de producción, volatilidad de precios y competencia por parte de productos importados con menor precio, perjudicando a las economías rurales (Anderson, 2018). Manuel Chiriboga (2015) explica que los procesos de liberalización e integración comercial tienen un impacto negativo en los niveles de bienestar y empleo, afectando los niveles de desigualdad y pobreza en zonas rurales.

En el Ecuador, la mayor parte de las políticas son orientadas hacia los agronegocios. Como resultado se obtiene una mayor diferenciación y polarización campesina, con desigualdad en el control de recursos como la tierra y el agua. Es así como pequeños productores, campesinos sin tierra, mujeres y madres rurales solas resultan acogidas en políticas de asistencia y no de desarrollo (Carrión y Herrera, 2012).

La AFC se ha visto estructuralmente empobrecida, lo que se relaciona con el bajo nivel de desarrollo económico y social alcanzado en las zonas rurales del Ecuador, siendo ésta catalogada muchas veces como una «deuda histórica», aunque sin lograr una acción efectiva. En este sentido, se vuelve relevante explorar de manera específica los niveles de empobrecimiento de la AFC a fin de generar nueva evidencia empírica que sirva de base para la generación de estrategias de erradicación de la pobreza. 
Con estos antecedentes, en este artículo se responde a las siguientes preguntas de investigación: ¿Cuál es la diferencia entre la tasa de pobreza multidimensional de los hogares de la AFC y los demás hogares del país? y ¿Cuáles son los principales determinantes de pobreza multidimensional de la AFC?

\section{REVISIÓN DE LITERATURA}

La pobreza indica una condición de privación o carencia. Ésta puede ubicarse ya sea en términos monetarios de ingreso o consumo; pero también en cuanto a insatisfacción de necesidades básicas, o bien como privación de libertades, capacidades o incluso de derechos. Si bien hay múltiples definiciones y formas de medición o aproximación, es el cálculo de la pobreza multidimensional la que ha tomado fuerza en los últimos años.

Desde el enfoque de derechos, la pobreza multidimensional se define como el incumplimiento del derecho humano de una persona a una serie de capacidades, recursos, opciones, seguridad, entre otros elementos relacionados con el ejercicio de derechos (Kumar, 2017). La medición de la pobreza requiere, entonces, identificar un conjunto de privaciones desde una proyección normativa.

Desde una perspectiva de desarrollo dual, existen sectores económicos y áreas que presentan menores niveles de productividad, pero que, además, quedan excluidas de servicios, acceso a mercados y disponibilidad de tecnología. Éste es el caso de las áreas rurales de países como el Ecuador, donde las personas presentan carencia de oportunidades básicas, empoderamiento y seguridad (Okidegbe, 2001). La pobreza rural se caracteriza por exclusión socioeconómica y discriminación basada en temas de género y etnia. Incluso presenta limitado acceso a los servicios básicos y los niveles de ingreso se encuentran por debajo de la canasta de bienes y servicios básicos (Chonchol, 2008).

En el caso de la educación, la escasez de recursos dificulta el acceso a ésta en el medio rural. Uno de los efectos de la pobreza en la educación es el abandono escolar, por falta de recursos económicos o por inserción al mercado laboral (Centro Latinoamericano para el Desarrollo Rural y Fondo Internacional de Desarrollo Agrícola [RIMISIP/FIDA], 2015). En cuanto a trabajo y seguridad social, la agricultura y ganadería son actividades en las cuales se emplean mano de obra infantil y adolescente (Naciones Unidas para la Infancia e Instituto Nacional de Estadística y Censos [UNICEF/INEC], 2015). Adicionalmente existen privaciones en términos de acceso a servicios de salud y agua segura que generan un círculo de reproducción de la pobreza (Ministerio de Salud Pública del Ecuador, 2018).

La AFC es una forma de organización productiva gestionada y dirigida por una familia. Es una forma de vida y de producción, caracterizada por la propiedad, trabajo familiar, control del proceso y técnica de producción, semillas nativas, entre otros (Herrera, 2021). Garner y De la O Campos (2014) mencionan tres elementos característicos de la AFC. Primero, las explotaciones agrícolas presentan un predominio de trabajo con base en el núcleo familiar. Segundo, el jefe o la jefa de hogar administra la unidad económica-productiva. Tercero, el nivel de explotación o producción es relativamente pequeño, en relación con el contexto del país.

Los agricultores familiares son un grupo que se ha visto afectado por la pobreza rural. En estas condiciones se da un proceso de empobrecimiento estructural de la AFC. Empobrecimiento que se observa en falta de ingresos y recursos productivos, limitación de oportunidades, exclusión social y vulnerabilidad, 
limitada participación política, entre otros elementos que acrecientan su vulnerabilidad, y estructuralizan la desigualdad frente a otros grupos poblacionales.

\section{METODOLOGÍA}

El cálculo de la pobreza multidimensional se realiza utilizando datos de las rondas de diciembre de 2009 a 2019, ${ }^{1}$ de la Encuesta Nacional de Empleo, Desempleo y Subempleo (ENEMDU) del INEC. La identificación de los hogares que corresponden a la AFC se establece en base a cuatro criterios: 1 . residencia en el sector rural; 2. rama de actividades agrícola, pecuaria, silvicultura, pesca y acuicultura; 3. categoría de ocupación como jornalero o peón, cuentapropista, trabajo no remunerado, o ayudante no remunerado; y 4. sitio o lugar de trabajo en local como patrono, local propio o arrendado, vivienda, finca o terreno propio, finca, terreno o establecimiento comunal, o en desplazamiento.

Por su parte, el cálculo del índice de pobreza multidimensional (IPM), se construye siguiendo la metodología de Alkire y Foster (2007, 2011), siendo el método oficial en el Ecuador y el más utilizado para la medición de la pobreza multidimensional, de manera que se garantiza una comparabilidad metodológica. Según el INEC (2015a), se utilizan cuatro dimensiones y doce indicadores.

El cálculo se realiza en dos pasos: primero, se emplea el método de corte dual en que se determina si hay privación en cada indicador; y segundo, se realiza un conteo de las privaciones considerando el umbral de pobreza multidimensional $k$. De esta manera se obtienen las siguientes medidas:

a. Tasa de pobreza multidimensional o tasa de recuento $(\mathrm{H})$ : se obtiene dividiendo el número de hogares identificados como pobres por el número total de hogares en la sociedad, representado como $H=q / n$. Donde q es el número de pobres identificados utilizando el enfoque de corte dual. Esta tasa corresponde al porcentaje de hogares que tienen privaciones en una tercera parte o más $(\mathrm{k}>=33,3 \%)$ de los indicadores ponderados.

b. Intensidad o proporción promedio de privación entre pobres (A): se calcula sumando la proporción del total de privaciones que sufre cada hogar pobre y dividiéndola por el número total de hogares pobres, es decir, es el porcentaje promedio de privaciones que tienen las hogares que son pobres multidimensionales, representado de la siguiente manera:

$$
A=\frac{\sum_{j=1}^{k} c(k)}{d q}
$$

En donde $\mathrm{c}(\mathrm{k})$ es el vector censurado de recuento de privaciones, si $\mathrm{ci} \geq \mathrm{k}$, entonces $\mathrm{c}(\mathrm{k})=\mathrm{ci}$, o el recuento de privaciones del hogar $\mathrm{i}$; $\mathrm{y}$ si $\mathrm{ci}<\mathrm{k}$, entonces $c(k)=0$ (Alkire y Foster, 2011).

c. Tasa de pobreza extrema multidimensional (TPEM): corresponde al porcentaje de hogares que tienen privaciones en al menos la mitad de los indicadores ponderados $(\mathrm{k}>=50 \%)$.

d. Tasa de recuento ajustada o índice de pobreza multidimensional (M0): se representa mediante la fórmula $M O=I P M=H^{*} A$. El IPM es un índice acotado entre 0 y 1 , donde 1 significa que todos los hogares son pobres en todos los indicadores y 0 en caso de que ningún hogar sea pobre multidimensional (INEC, 2015a).

Una vez calculado el IPM se determina la contribución de cada una de las dimensiones e indicadores. Para relacionar las dimensiones de la pobreza multidimensional 
en los hogares de la AFC, se realiza un análisis de superposición de privaciones. Primero, se realiza el análisis de privaciones individuales, en el cual se estima la proporción de recuento de privación para cada indicador y dimensión. A continuación, se realiza el análisis de privación superpuesta múltiple, en el cual se calcula para cada hogar, el número de dimensiones en las que está privado. Se calcula la proporción de hogares privados en $0,1,2, \ldots$, d dimensiones. Para el recuento de privaciones se utiliza la siguiente fórmula.

$$
D i=\sum_{j=1}^{d} y_{i}
$$

Donde Di es el número total de dimensiones en las que se priva a cada hogar. Si yj $=1$, el hogar i está privado en la dimensión j; yj = 0 si el hogar i no está privado en la dimensión j.

El análisis de las superposiciones de privaciones permite examinar qué tipo de privaciones experimentan los hogares simultáneamente, las superposiciones de privación se realizan combinando las cuatro dimensiones en un diagrama de Venn, el cual permite una representación del grado de privaciones (De Neubourg, Chai y De Milliano, 2012). También, se realiza una matriz de redundancia o de traslape entre los indicadores y dimensiones planteada por Alkire et al. (2015).

Por último, mediante un modelo probabilístico (Probit) se identifica los determinantes de la pobreza multidimensional en los hogares de la AFC. El modelo se estructura de la siguiente manera:

$$
\operatorname{Pr}\left(Y_{i}=1 \mid X\right)=P(Z i \leq \beta 0+\beta 1 X i)=F(\beta 0+\beta 1 X i) .
$$

En donde, $\mathrm{P}(\mathrm{Y}=1 \mid \mathrm{X})$ significa la probabilidad de que un hogar cumpla con las condiciones de encontrarse en situación de pobreza dado las variables explicativas. En este caso, Y identifica con el valor de 1 a los hogares en situación de pobreza multidimensional y con valor de 0 a los no pobres multidimensionalmente. $\mathrm{Xi}$ será el género junto con factores sociodemográficos y una variable de contexto (región). Zi es la variable normal estandarizada; es decir, Z N $\left(0, \sigma^{2}\right)$. Después, se utilizan efectos marginales de los indicadores sobre la probabilidad de que sean pobres multidimensionales para interpretar los resultados. Esto se realizará para jefatura de hogar.

\section{RESULTADOS}

A diciembre de 2019 se identifican aproximadamente 1,8 millones de personas que pertenecen a la AFC, que componen cerca de 375.000 hogares, en el Ecuador. El ingreso promedio real del jefe o jefa de hogar que pertenece a este sistema de producción alcanzó los USD 256,72 mensuales. Solo el 29,8\% tenían acceso a agua por red pública.

La tabla 1 muestra que la tasa de pobreza multidimensional en la AFC pasó del 94,2\% en 2009 al 86,2\% en 2019, representando una disminución del 8,5\% en el caso de la AFC; mientras que a nivel nacional la reducción fue del 31,3\% y a nivel rural fue de 19,1\%, lo que evidencia que la brecha de empobrecimiento ( $i$. e. desigualdad) ha aumentado en los últimos 10 años (ver Tabla 1).

Al comparar las tasas a nivel nacional (30,5\%) y rural (63,5\%), se observa que el nivel de empobrecimiento rural duplica el nivel nacional a 2019, y que representa, además, un porcentaje superior al de la pobreza por ingresos que en el área rural alcanzó el 41,8\%. En el caso de la AFC la situación es aún más 
Tabla 1. Evolución de la tasa de pobreza multidimensional (TPM), intensidad (A), índice de pobreza multidimensional (IPM) y tasa de pobreza extrema multidimensional (TPEM), a nivel nacional (N), rural (R) y de la agricultura familiar campesina (AFC) (2009-2019) (en porcentaje).

\begin{tabular}{|c|c|c|c|c|c|c|c|c|c|c|c|c|}
\hline \multirow{2}{*}{ AÑO } & \multicolumn{3}{|l|}{ TPM } & \multicolumn{3}{|l|}{ A } & \multicolumn{3}{|c|}{ TPEM } & \multicolumn{3}{|l|}{ IPM } \\
\hline & $\mathrm{N}$ & $\mathrm{R}$ & $\mathrm{AFC}$ & $\mathrm{N}$ & $\mathrm{R}$ & $\mathrm{AFC}$ & $\mathrm{N}$ & $\mathrm{R}$ & $\mathrm{AFC}$ & $\mathrm{N}$ & $\mathrm{R}$ & $\mathrm{AFC}$ \\
\hline 2009 & 44,4 & 78,5 & 94,2 & 50,7 & 54,1 & 59,9 & 22,2 & 47,9 & 73,0 & 22,5 & 42,5 & 56,4 \\
\hline 2010 & 39,4 & 74,7 & 92,4 & 49,9 & 53,1 & 59,6 & 18,8 & 43,6 & 71,8 & 19,7 & 39,7 & 55,1 \\
\hline 2011 & 35,6 & 72,1 & 90,7 & 48,9 & 51,5 & 56,1 & 15,8 & 38,6 & 60,5 & 17,4 & 37,1 & 50,8 \\
\hline 2012 & 32,6 & 63,3 & 87,4 & 48,6 & 50,8 & 56,2 & 14,1 & 35,1 & 59,7 & 15,8 & 34,7 & 49,2 \\
\hline 2013 & 32,7 & 63,2 & 84,9 & 47,7 & 50,6 & 56,3 & 13,2 & 32,5 & 58,8 & 15,6 & 32,0 & 47,8 \\
\hline 2014 & 31,2 & 58,7 & 86,3 & 47,2 & 50,1 & 55,2 & 11,9 & 29,1 & 55,5 & 14,7 & 29,4 & 47,7 \\
\hline 2015 & 28,8 & 57,0 & 84,8 & 47,3 & 50,3 & 56,2 & 10,9 & 28,2 & 56,2 & 13,6 & 28,7 & 47,6 \\
\hline 2016 & 28,8 & 52,9 & 81,6 & 46,7 & 49,8 & 55,1 & 10,2 & 24,4 & 50,4 & 13,4 & 26,4 & 45,0 \\
\hline 2017 & 28,3 & 56,1 & 83,2 & 47,2 & 50,6 & 56,0 & 10,5 & 27,8 & 54,6 & 13,4 & 28,4 & 46,5 \\
\hline 2018 & 30,4 & 60,5 & 82,4 & 47,6 & 50,7 & 55,5 & 11,9 & 30,5 & 53,4 & 14,5 & 30,7 & 45,7 \\
\hline 2019 & 30,5 & 63,5 & 86,2 & 47,7 & 50,8 & 56,2 & 12,0 & 32,0 & 54,6 & 14,5 & 32,3 & 48,4 \\
\hline
\end{tabular}

Fuente: sobre la base de la Encuesta Nacional de Empleo, Desempleo y Subempleo (ENEMDU) de las rondas de diciembre.

crítica, al alcanzar una tasa de pobreza multidimensional del 86,2\% (equivalente a 323.000 hogares) (ver Figura 1).

A nivel nacional, el IPM llegó a 14,5 puntos siendo la mayor privación el desempleo o empleo inadecuado. En la zona rural el índice al 2019 fue de 32,3 y la mayor privación es sin servicio de agua por red pública. El índice alcanzó los 48,4 puntos para la AFC, en 2019, siendo las cinco principales carencias las relacionadas a los indicadores de: sin servicio de agua por red pública (17,8\%), desempleo o empleo inadecuado (14,8\%), logro educativo incompleto (12,9\%), no contribución al sistema de pensiones (12,1\%) y déficit habitacional (9,3\%). Como se puede observar, estos cinco indicadores permanecen en los primeros lugares a lo largo del tiempo (ver Figura 1).

En la tabla 2, se observa el porcentaje de hogares con privaciones por dimensión e indicador. En ese sentido, la dimensión de trabajo y seguridad social destaca como la de mayor privación en los hogares del Ecuador. A nivel nacional y rural, se obtiene una reducción de 6,3 y 2 puntos porcentuales (p. p.), respectivamente, mientras que en la AFC no presenta cambios en el periodo analizado. En esta última se observa, en las otras dimensiones, una reducción en el porcentaje de hogares con privaciones (ver Tabla 2).

Al analizar la prevalencia de carencias por indicador, se observa que al 2019, el 10,2\% de los hogares tiene al menos una persona entre 18 a 29 años que no tiene acceso a educación superior por razones económicas, el 99,6 \% de los hogares tiene al menos una persona de 18 años o más que se encuentra en desempleo o tiene un empleo inadecuado y el 36,6\% de los hogares tienen por lo menos una persona entre 5 a 17 años que ejerce algún tipo de trabajo.

Así mismo, el 70,2\% de los hogares no tienen servicio de agua por red pública. El 81,5\% de los hogares tiene al menos un miembro de la familia entre 18 y 64 años que no han terminado su educación, mientras que el 78,0\% de las familias tienen al menos una persona ocupada de 15 años o más que no aporta a ningún tipo de seguridad social, el 80,7 \% de los hogares presentan déficit habitacional, es decir, que pueden tener carencias habitacionales en la estructura (cualitativo) o necesitan reemplazar una unidad habitacional (cuantitativo). Finalmente, 
Figura 1. Tasa de pobreza multidimensional y descomposición del índice Evolución de la tasa de pobreza multidimensional y tasa de pobreza por ingreso (2009-2019) (en porcentaje).

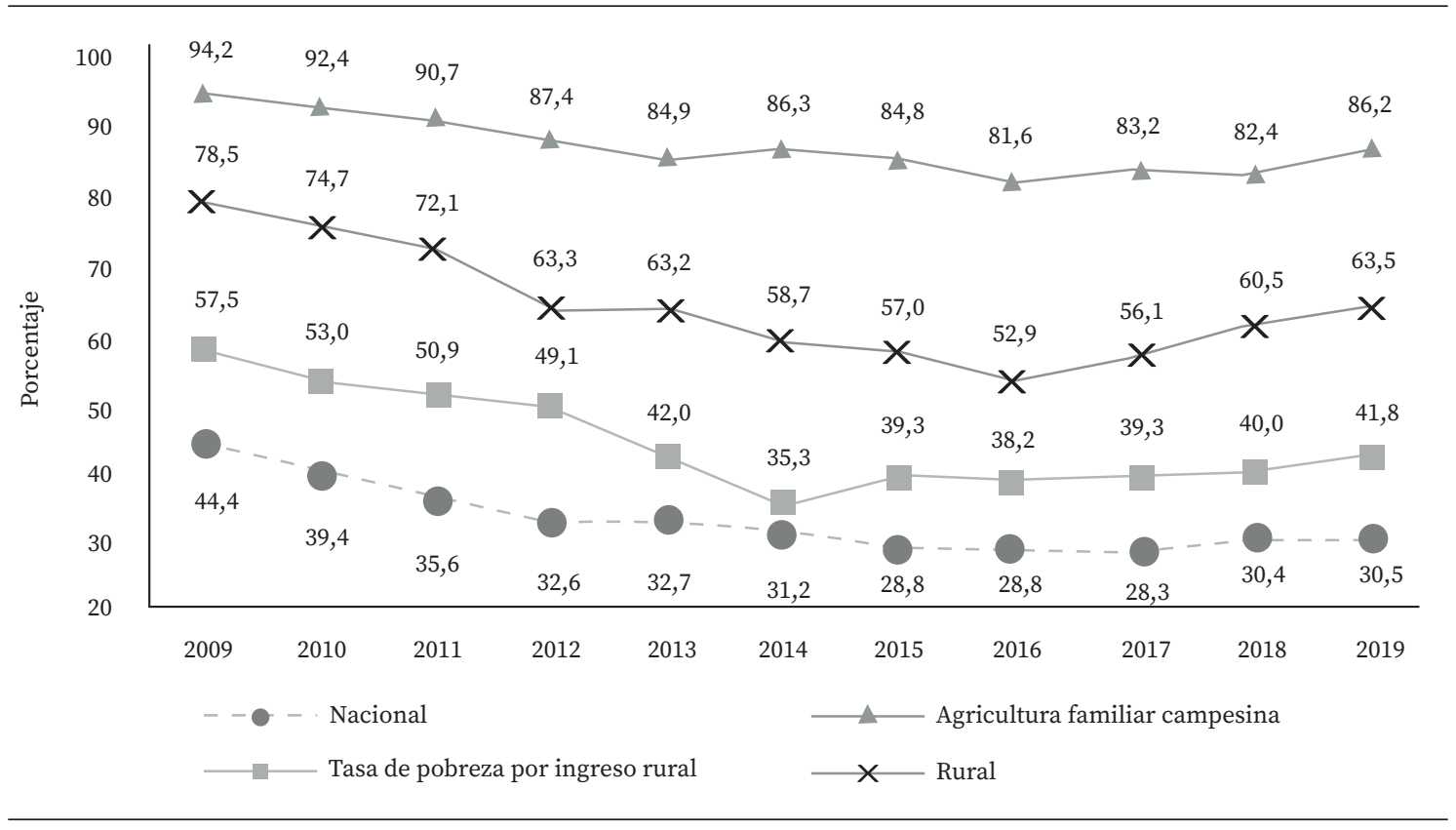

Fuente: sobre la base de la ENEMDU de las rondas de diciembre.

Tabla 2. Tasas de privaciones de los hogares por dimensión e indicador en la agricultura familiar campesina (2009-2019) (en porcentaje)

\begin{tabular}{|c|c|c|c|c|c|c|c|c|c|c|c|c|c|}
\hline \multirow[t]{2}{*}{ Dimensión } & \multicolumn{2}{|c|}{$\mathrm{N}$} & \multicolumn{2}{|c|}{$\mathrm{R}$} & \multicolumn{2}{|c|}{$\mathrm{AFC}$} & \multirow{2}{*}{ 莺 } & \multicolumn{2}{|c|}{$\mathrm{N}$} & \multicolumn{2}{|c|}{$\mathrm{R}$} & \multicolumn{2}{|c|}{$\mathrm{AFC}$} \\
\hline & 2009 & 2019 & 2009 & 2019 & 2009 & 2019 & & 2009 & 2019 & 2009 & 2019 & 2009 & 2019 \\
\hline \multirow[t]{3}{*}{ Educación } & 61,7 & 51,8 & 79,4 & 71,7 & 90,2 & 84,1 & 1 & 14,0 & 8,6 & 20,6 & 12,8 & 28,7 & 18,9 \\
\hline & & & & & & & 2 & 6,1 & 6,3 & 4,6 & 7,8 & 4,8 & 10,2 \\
\hline & & & & & & & 3 & 56,6 & 47,6 & 77,2 & 69,1 & 88,8 & 81,5 \\
\hline \multirow{3}{*}{$\begin{array}{l}\text { Trabajo y } \\
\text { seguridad social }\end{array}$} & 83,0 & 76,7 & 89,9 & 87,9 & 99,9 & 100 & 4 & 7,7 & 5,9 & 14,4 & 16,0 & 30,8 & 36,6 \\
\hline & & & & & & & 5 & 65,1 & 64,1 & 78,5 & 81,0 & 97,1 & 99,6 \\
\hline & & & & & & & 6 & 71,6 & 63,6 & 72,7 & 67,6 & 82,0 & 78,0 \\
\hline \multirow{2}{*}{$\begin{array}{l}\text { Salud, agua y } \\
\text { alimentación }\end{array}$} & 36,7 & 23,1 & 74,1 & 59,7 & 85,9 & 73,8 & 7 & 12,5 & 6,1 & 24,4 & 13,3 & 39,1 & 27,4 \\
\hline & & & & & & & 8 & 31,0 & 20,3 & 67,4 & 57,0 & 77,4 & 70,2 \\
\hline \multirow{4}{*}{$\begin{array}{l}\text { Hábitat, } \\
\text { vivienda } \\
\text { y ambiente sano }\end{array}$} & 68,7 & 56,5 & 91,9 & 81,9 & 85,9 & 91,2 & 9 & 16,1 & 10,4 & 20,9 & 12,5 & 24,8 & 17,7 \\
\hline & & & & & & & 10 & 56,9 & 48,4 & 79,6 & 73,0 & 83,1 & 80,7 \\
\hline & & & & & & & 11 & 31,5 & 19,1 & 47,2 & 26,3 & 58,3 & 37,8 \\
\hline & & & & & & & 12 & 28,5 & 15,2 & 68,1 & 47,9 & 85,2 & 64,9 \\
\hline
\end{tabular}

Fuente: sobre la base de la ENEMDU de las rondas de diciembre.

el 27,4\% se encuentran en pobreza extrema, es decir, que su ingreso per cápita familiar es inferior al de la línea de pobreza.

A nivel nacional y rural existen hogares que no tienen privación en las dimensiones. Las distribuciones a nivel nacional en el 2009 y 2019 alcanzan el punto máximo en tres dimensiones privadas y en la zona rural eran cuatro dimensiones privadas. El porcentaje de hogares privados en las cuatro dimensiones, en el 2019 son $14,8 \%$ y $39,4 \%$ a correspondencia. 
Con respecto a la AFC, se debe señalar que ningún hogar se encuentra libre de privaciones. En 2009 el porcentaje de familias que se encontraban privadas en 4 dimensiones eran el 76,5\%, porcentaje que se reduce hasta el 60,2\% en 2019. En el caso del porcentaje de hogares que están privados en una, dos y tres dimensiones se observa un aumento de 1,2; 4,8 y 10,4 puntos porcentuales, respectivamente.

En el análisis de la superposición de las dimensiones realizado para los hogares privados y no privados. Se obtiene que la AFC llegaría a cuadruplicar la proporción de privación superpuesta en todas las dimensiones (60,2\%) en comparación con los hogares a nivel nacional $(14,8 \%)$ y casi duplica el porcentaje de la zona rural $(39,4 \%)$.

Las cifras muestran que alrededor del 60,2\% de hogares de la AFC tienen mayor probabilidad de estar privados en todas las dimensiones mencionadas. Se obtiene que 5000 hogares se encuentran privados en trabajo y seguridad social $(1,4 \%)$. Alrededor de 11.000 hogares se encuentran privados en las dimensiones de educación y trabajo simultáneamente (3,0\%), mientras que 38.000 hogares son privados en trabajo y hábitat (10,2\%). Para el caso de privación simultánea en tres dimensiones: hábitat, educación y trabajo se observa a 65.000 hogares $(17,4 \%)$.

A nivel de la AFC se obtiene que trabajo y seguridad social es la dimensión con alta redundancia, es decir, que si en un hogar se presenta un miembro que esté privado en dicha dimensión es muy probable que se encuentre privado en las demás dimensiones. En el caso de los indicadores, el desempleo o empleo inadecuado tiene mayor redundancia (ver Anexos 1 y 2).

La AFC pertenece a la zona rural, en donde los indicadores de empobrecimiento siguen siendo relativamente altos. Asimismo, las mediciones multidimensionales muestran niveles alarmantes de población en condiciones de pobreza, lo que es consistente con estudios regionales (por ejemplo, Maletta, 2011 y FAO, 2018c). Al obtener el índice de pobreza multidimensional tanto en la AFC como a nivel nacional se observa que éste se ha reducido a lo largo del periodo de análisis (2009-2019); sin embargo, la brecha en comparación con el nivel nacional e incluso con el total del área rural se ha incrementado. Asimismo, el número de privaciones (intensidad de la pobreza) es alto, lo que da cuenta de factores estructurales de empobrecimiento en la AFC, constituyendo un desafío para el cumplimiento de varias de las principales metas de la Agenda 2030 de los Objetivos de Desarrollo Sostenible (ODS) que establecen reducir la pobreza en todas sus formas y la proporción de personas que viven en estas condiciones, así como garantizar que los pobres tengan los mismos derechos, y aumentar el acceso seguro y equitativo a varios servicios, en especial de agricultores familiares y mujeres rurales.

Para reducir la pobreza en la AFC, además de mejorar los ingresos de estos hogares mediante mejoras de productividad agrícola y acceso a mercados, se debe contar con servicios básicos, oportunidades y acceso a educación y vivienda digna (FAO, 2018b). En esta línea, el caso del Ecuador, al descomponer el IPM se encuentra que los factores que explican más de la mitad de la pobreza son: carencia de servicio de agua segura, desempleo o empleo inadecuado, logro educativo incompleto, no contribución al sistema de pensiones y déficit habitacional. La privación de acceso a agua segura se ubica en el primer lugar, ya que más del $70 \%$ de los hogares en la AFC no tienen este servicio. Además, históricamente la acción pública se ha enfocado en los productores medianos y grandes, dejando de lado a la pequeña producción, así como a familias campesinas y mujeres rurales, por lo que no se han resuelto las privaciones en esta población.

Una de las contribuciones que se da en cuanto al análisis de interseccionalidad de privaciones, es la metodología de De Neubourg et al. (2012), la cual permite observar que la mayoría de los hogares se encuentran privados en las cuatro dimensiones del IPM. Además, si el hogar está privado en alguna de las cuatro 
dimensiones es muy probable que esté privado también en 2 o 3 dimensiones conjuntamente. Con estos resultados se puede decir que existe mayor vulnerabilidad porque se presentan mayor número de privaciones que actúan a la vez.

La dimensión con mayor redundancia es la de trabajo y seguridad social, lo que da cuenta de una estructura económica que excluye y empobrece a la AFC. La agricultura es una de las principales fuentes de empleo e ingreso para la zona rural (Martínez, 2013), pero ha mostrado una tendencia hacia el empleo temporal en lugar del permanente. Los asalariados agrícolas presentan mayores tasas de trabajadores que no tienen contrato ni previsión social, al mismo tiempo ha aumento la subcontratación de mano de obra, la cual está asociada con niveles altos de desprotección para trabajadores (Schejtman, 2008). Para el caso de los jóvenes y de las mujeres rurales tener empleo no es equivalente a un trabajo decente y mucho menos los ingresos pueden solventar las condiciones para el desarrollo (FAO, 2016; FAO, 2018c).

Se realiza una caracterización de los factores socioeconómicos, donde se toman 27.235 jefes de hogar de la AFC del 2009 al 2019. Solo el 16\% de los hogares presenta jefatura femenina y del total de los hogares 19,4\% son monoparentales. Además, cerca de 2673 jefes de hogar tienen un trabajo secundario, donde las actividades agrícolas representan el 81,5\% mientras que el 18,5\% son actividades no agrícolas. El 77,4\% de los jefes de hogar son mayores de 40 años. Se utiliza la variable región, ya que se observa que el mayor porcentaje de hogares de la AFC se encuentran en la Sierra. El número de dependientes en el hogar en promedio se encuentra entre dos personas (ver Tabla 3 ).

A través de la aplicación de un modelo probabilístico se encuentra que la variable género (jefatura femenina) no es una variable con significancia estadística. Según Alemu, Bewket, Zeleke, Assefa y Trutman (2011), el género es una variable que puede tener diferentes efectos basado en el contexto socioeconómico. En varios estudios se obtiene que los hogares con jefatura masculina tienen mayor probabilidad de estar en condición de pobreza que los hogares encabezados por mujeres, una de las razones detrás es porque existe una infrarrepresentación de hogares con jefatura femenina (Okeke-Agulu, Arene y Noble, 2014; Majeed y Malik, 2015; Ibitola, Fasakin, Popoola y Olajide, 2019; Sadiq, Singh, Ahmad, Lawal y Kabeer, 2019). Como ya se mencionó, los hogares encabezados por mujeres representan un porcentaje menor. En cuestión de género, la desigualdad causada por la división sexual del trabajo ha logrado que se ignore el aporte de la mujer en la producción. Las mujeres rurales son las más afectadas por la pobreza, ya que las características patriarcales que tiene la sociedad ocasionan mayor presión, discriminación, sobrecarga laboral y explotación hacia ellas (Carrión y Herrera, 2012; FAO, 2018c; Flores, s. f.) (ver Tabla 4).

Al mismo tiempo, se obtiene que los determinantes de la pobreza multidimensional son variables sociodemográficas. Cabe aclarar que es una relación de correlación, por lo que no implicaría causalidad. Variables como la escolaridad y número de dependientes en el hogar influyen en la probabilidad de empobrecimiento. Se obtiene que la escolaridad es una variable significativa y la probabilidad de estar en condiciones de pobreza se reduce, lo cual concuerda con estudios como Apata, Igbalajobi y Awoniyi (2010), Sudhakara y Nega (2013), Majeed y Malik (2015) y Adepoju (s. f.). La educación del jefe de hogar es beneficiosa para superar la prevalencia de pobreza multidimensional de manera individual, incluso para los otros miembros de la familia (Majeed y Malik, 2015). En el caso de los dependientes en el hogar, el aumento de una unidad adicional puede asociarse con un aumento en la probabilidad de pobreza del $3 \%$.

En el modelo realizado para el nivel nacional se obtiene que la variable AFC y el área rural son variables que afectan de manera directa a la probabilidad de encontrarse en pobreza multidimensional. Por su parte, el modelo en el ámbito rural muestra que la AFC puede estar relacionada con una mayor probabilidad de presentar condiciones de pobreza. 
Tabla 3. Características socioeconómicas a nivel de jefatura de hogares (2009-2019) (en porcentaje)

\begin{tabular}{|c|c|c|c|c|c|c|c|}
\hline \multirow{2}{*}{$\begin{array}{l}\text { Variable } \\
\text { Frecuencia }\end{array}$} & & \multicolumn{2}{|l|}{ Nacional } & \multicolumn{2}{|l|}{ Rural } & \multicolumn{2}{|l|}{ AFC } \\
\hline & & Frecuencia & $\%$ & Frecuencia & $\%$ & Frecuencia & $\%$ \\
\hline \multirow{2}{*}{ Género } & Hombre & 131.545 & 73,5 & 56.723 & 78,2 & 22.775 & 83,6 \\
\hline & Mujer & 47.366 & 26,5 & 15.797 & 21,8 & 4.460 & 16,3 \\
\hline \multirow{2}{*}{ Estructura de hogar } & Monoparentales & 65.433 & 37,1 & 23.915 & 33,4 & 5.216 & 19,4 \\
\hline & Biparentales & 111.261 & 62,9 & 47.667 & 66,6 & 21.686 & 80,6 \\
\hline \multirow{2}{*}{$\begin{array}{l}\text { Participación } \\
\text { en actividades }\end{array}$} & Agrícolas & 4.463 & 49,5 & 3.676 & 71,9 & 2,179 & 81,5 \\
\hline & No agrícolas & 4.563 & 50,5 & 1.439 & 28,1 & 494 & 18,5 \\
\hline \multirow{6}{*}{$\begin{array}{l}\text { Años del jefe } \\
\text { de hogar }\end{array}$} & Menor de 20 & 1.500 & 0,8 & 506 & 0,7 & 80 & 0,3 \\
\hline & $21-30$ & 18.286 & 10,2 & 6.609 & 9,1 & 1.639 & 6,0 \\
\hline & $31-40$ & 34.572 & 19,3 & 13.374 & 18,4 & 4.447 & 16,3 \\
\hline & $41-50$ & 38.945 & 21,8 & 15.106 & 20,8 & 6.209 & 22,8 \\
\hline & $51-60$ & 34.598 & 19,3 & 13.363 & 18,4 & 5.852 & 21,5 \\
\hline & 61 a más & 51.010 & 28,5 & 23.562 & 32,5 & 9.008 & 33,1 \\
\hline \multirow{3}{*}{ Región } & Costa & 64.247 & 36,2 & 21.513 & 29,9 & 6.392 & 23,5 \\
\hline & Sierra & 91.171 & 51,3 & 38.315 & 53,1 & 15.249 & 56,1 \\
\hline & Amazonía & 22.322 & 12,5 & 12.314 & 17,0 & 5.521 & 20,3 \\
\hline $\begin{array}{l}\text { Horas trabajo } \\
\text { semanal }\end{array}$ & & Media & 41,3 & Media & 39,3 & Media & 39,2 \\
\hline Escolaridad & & Media & 8,9 & Media & 6,5 & Media & 5,9 \\
\hline $\begin{array}{l}\text { Dependientes en } \\
\text { el hogar }\end{array}$ & & Media & 1,8 & Media & 2,0 & Media & 2,4 \\
\hline
\end{tabular}

Fuente: sobre la base de la ENEMDU de las rondas de diciembre.

Tabla 4. Resultados del modelo por jefatura de hogar (2009-2019) (en porcentaje)

\begin{tabular}{|c|c|c|c|c|c|c|c|c|c|c|c|c|}
\hline \multirow[b]{2}{*}{ Variable } & \multicolumn{4}{|c|}{ Nacional } & \multicolumn{4}{|l|}{ Rural } & \multicolumn{4}{|l|}{ AFC } \\
\hline & Coef. & $\begin{array}{l}\text { Ef. } \\
\text { marginales }\end{array}$ & $\begin{array}{l}\text { Error } \\
\text { estándar }\end{array}$ & $\mathbf{P}>|\mathbf{z}|$ & Coef. & $\begin{array}{l}\text { Ef. } \\
\text { marginales }\end{array}$ & $\begin{array}{l}\text { Error } \\
\text { estándar }\end{array}$ & $\mathbf{P}>|\mathbf{z}|$ & Coef. & $\begin{array}{l}\text { Ef. } \\
\text { marginales }\end{array}$ & $\begin{array}{l}\text { Error } \\
\text { estándar }\end{array}$ & $\mathbf{P}>|\mathbf{z}|$ \\
\hline $\begin{array}{l}\text { Género } \\
\text { (mujer) }\end{array}$ & $-0,106$ & $-0,024$ & 0,061 & 0,082 & $-0,234$ & $-0,060$ & 0,080 & 0,004 & $-0,049$ & $-0,009$ & 0,167 & 0,766 \\
\hline $\mathrm{AFC}$ & 0,881 & 0,204 & 0,488 & 0,000 & 0,954 & 0,246 & 0,050 & 0,000 & - & - & - & - \\
\hline Rural & 0,661 & 0,153 & 0,0432 & 0,000 & - & - & - & - & - & - & - & - \\
\hline $\begin{array}{l}\text { Hogares } \\
\text { monoparentales }\end{array}$ & 0,010 & 0,002 & 0,057 & 0,860 & 0,142 & 0,036 & 0,074 & 0,057 & $-0,121$ & $-0,024$ & 0,155 & 0,432 \\
\hline $\begin{array}{l}\text { Participación en } \\
\text { actividades no }\end{array}$ & & & & & & & & & & & & \\
\hline agrícolas & 0,000 & 0,000 & 0,000 & 0,322 & 0,000 & 0,000 & 0,000 & 0,140 & $-0,067$ & $-0,013$ & 0,088 & 0,443 \\
\hline $\begin{array}{l}\text { Edad del jefe } \\
\text { de hogar }\end{array}$ & 0,029 & 0,006 & 0,008 & 0,001 & 0,024 & 0,006 & 0,010 & 0,024 & 0,021 & 0,004 & 0,017 & 0,222 \\
\hline Región Costa & 0,664 & 0,154 & 0,316 & 0,036 & 0,920 & 0,238 & 0,400 & 0,021 & 1,039 & 0,206 & 0,663 & 0,117 \\
\hline Amazonia & 0,384 & 0,089 & 0,142 & 0,007 & 0,284 & 0,073 & 0,179 & 0,113 & 0,240 & 0,047 & 0,248 & 0,334 \\
\hline Edad2 & $-0,000$ & $-0,000$ & 0,000 & 0,000 & -0.000 & $-0,000$ & 0,000 & 0,000 & $-0,000$ & $-0,000$ & 0,000 & 0,040 \\
\hline $\begin{array}{l}\text { Horas de trabajo } \\
\text { semanal }\end{array}$ & $-0,008$ & $-0,002$ & 0,001 & 0,000 & -0.005 & $-0,001$ & 0,001 & 0,000 & $-0,001$ & $-0,000$ & 0,002 & 0,587 \\
\hline Escolaridad & $-0,129$ & $-0,030$ & 0,004 & 0,000 & $-0,109$ & $-0,028$ & 0,006 & 0,000 & $-0,092$ & $-0,018$ & 0,010 & 0,000 \\
\hline $\begin{array}{l}\text { Dependientes } \\
\text { en el hogar }\end{array}$ & 0,167 & 0,389 & 0,012 & 0,000 & 0,115 & 0,029 & 0,015 & 0,000 & 0,155 & 0,030 & 0,024 & 0,000 \\
\hline cons & 0,395 & & 0,245 & 0,107 & 1,039 & & 0,303 & 0,001 & 1,999 & & 0,499 & 0,000 \\
\hline
\end{tabular}




\begin{tabular}{llll}
\hline & Nacional & Rural & AFC \\
Sensibilidad & 78,18 & 88,62 & 97,77 \\
Especificidad & 82,62 & 57,10 & 17,04 \\
Correctamente & 80,54 & 78,55 & 84,85 \\
clasificado & 178.911 & 72.520 & 27.235 \\
N & 8.008 & 4.470 & 2.238 \\
Observaciones & 0,3993 & 0,2654 & 0,1852 \\
\hline
\end{tabular}

Fuente: sobre la base de la ENEMDU de las rondas de diciembre.

\section{CONCLUSIONES}

El artículo analiza la pobreza multidimensional de los hogares de la AFC en Ecuador. Se encuentra que, si bien el índice de pobreza multidimensional se reduce entre 2009 y 2019, la brecha con relación al promedio nacional e incluso en la zona rural ha aumentado. Asimismo, se observa alta interseccionalidad entre privaciones; la mayor parte de hogares de la AFC se encuentran privados en las cuatro dimensiones; y la dimensión de mayor privación es la de trabajo y seguridad social.

Los determinantes de la pobreza son variables sociodemográficas como la escolaridad, que se relaciona de manera inversa a la probabilidad de pobreza multidimensional, al contrario del número de dependientes en el hogar que se relaciona con mayor probabilidad de pobreza multidimensional.

En este sentido, se encuentra que la ausencia de sistemas de protección social adecuados, sumado a la estructura económica de baja productividad y fallas de acceso a mercados en la agricultura, ganadería y pesca, son los factores estructurales de empobrecimiento de la AFC.

En términos de política pública es necesaria una visión multisectorial para mejorar la calidad de vida de los hogares de la AFC. Para la cual es necesario un grupo de políticas orientadas a la producción, sostenibilidad de recursos, igualdad de género, empleo adolescente, educación, provisión de servicios públicos, entre otros factores, dentro de estrategias integrales de desarrollo local y regional.

\section{NOTA FINAL}

1. La pobreza multidimensional en el Ecuador se evalúa desde una perspectiva de derechos, y se mide siguiendo la metodología de Alkire y Foster (2007, 2011), de acuerdo con lo establecido por el Instituto Nacional de Estadística y Censos ([INEC], 2015a). Las dimensiones que se evalúan son educación; trabajo y seguridad social; salud, agua y alimentación; y hábitat, vivienda y ambiente sano, que corresponden a una agrupación de los derechos del buen vivir establecidos en la Constitución. 
Anexo 1. Matriz de redundancia de dimensiones a nivel de los hogares (2009-2019) (en porcentaje)

$\begin{array}{lll}\text { Dimensiones Nacional } & \text { Rural }\end{array}$

\begin{tabular}{lllllllllllll}
\hline & $\mathrm{E}$ & $\mathrm{T}$ & $\mathrm{S}$ & $\mathrm{H}$ & $\mathrm{E}$ & $\mathrm{T}$ & $\mathrm{S}$ & $\mathrm{H}$ & $\mathrm{E}$ & $\mathrm{T}$ & $\mathrm{S}$ & $\mathrm{H}$ \\
Educación & 100 & & & & 100 & & & & 100 & & & \\
$\begin{array}{l}\text { Trabajo y } \\
\text { seguridad social }\end{array}$ & 85,88 & 100 & & & 89,80 & 100 & & & 99,87 & 100 & & \\
$\begin{array}{l}\text { Salud, agua y } \\
\text { alimentación }\end{array}$ & 76,65 & 86,60 & 100 & & 78,67 & 87,48 & 100 & & 88,20 & 99,84 & 100 & \\
$\begin{array}{l}\text { Hábitat, vivienda } \\
\text { y ambiente sano }\end{array}$ & 72,03 & 82,18 & 90,01 & 100 & 85,21 & 86,73 & 92,15 & 100 & 92,94 & 99,87 & 95,58 & 100 \\
\hline
\end{tabular}

Fuente: sobre la base de la ENEMDU de las rondas de diciembre.

Anexo 2. Matriz de redundancia de indicadores a nivel de los hogares (2009-2019) (en porcentaje)

\begin{tabular}{|c|c|c|c|c|c|c|c|c|c|c|c|c|}
\hline & \multicolumn{12}{|c|}{ Nacional } \\
\hline & 1 & 2 & 3 & 4 & 5 & 6 & 7 & 8 & 9 & 10 & 11 & 12 \\
\hline 1 & 100 & & & & & & & & & & & \\
\hline 2 & 9,6 & 100 & & & & & & & & & & \\
\hline 3 & 76,5 & 58,0 & 100 & & & & & & & & & \\
\hline 4 & 54,9 & 5,8 & 84,7 & 100 & & & & & & & & \\
\hline 5 & 73,7 & 81,1 & 74,0 & 87,9 & 100 & & & & & & & \\
\hline 6 & 76,7 & 74,8 & 74,5 & 86,7 & 78,5 & 100 & & & & & & \\
\hline 7 & 21,7 & 8,9 & 77,0 & 21,1 & 90,0 & 76,5 & 100 & & & & & \\
\hline 8 & 37,5 & 21,2 & 74,2 & 49,5 & 76,0 & 66,7 & 51,2 & 100 & & & & \\
\hline 9 & 30,7 & 15,6 & 79,3 & 29,0 & 75,0 & 78,9 & 30,6 & 37,0 & 100 & & & \\
\hline 10 & 65,0 & 53,2 & 64,8 & 71,2 & 70,9 & 68,3 & 75,9 & 77,5 & 75,6 & 100 & & \\
\hline 11 & 37,9 & 22,4 & 70,0 & 42,3 & 72,5 & 69,5 & 45,3 & 52,7 & 44,0 & 77,0 & 100 & \\
\hline \multirow[t]{3}{*}{12} & 31,6 & 15,2 & 72,9 & 47,5 & 77,5 & 66,1 & 46,2 & 69,3 & 28,8 & 76,6 & 48,3 & 100 \\
\hline & \multicolumn{12}{|c|}{ Rural } \\
\hline & 1 & 2 & 3 & 4 & 5 & 6 & 7 & 8 & 9 & 10 & 11 & 12 \\
\hline 1 & 100 & & & & & & & & & & & \\
\hline 2 & 13,1 & 100 & & & & & & & & & & \\
\hline 3 & 90,1 & 76,4 & 100 & & & & & & & & & \\
\hline 4 & 54,1 & 11,2 & 90,5 & 100 & & & & & & & & \\
\hline 5 & 82,9 & 86,6 & 81,6 & 91,8 & 100 & & & & & & & \\
\hline 6 & 77,8 & 76,3 & 80,8 & 83,9 & 86,1 & 100 & & & & & & \\
\hline 7 & 26,9 & 16,1 & 82,0 & 27,9 & 90,9 & 74,8 & 100 & & & & & \\
\hline 8 & 68,3 & 50,6 & 76,3 & 66,6 & 78,9 & 65,4 & 69,7 & 100 & & & & \\
\hline 9 & 33,9 & 16,4 & 91,0 & 30,4 & 84,3 & 26,3 & 33,1 & 70,2 & 100 & & & \\
\hline 10 & 78,2 & 67,2 & 74,2 & 76,7 & 78,9 & 71,4 & 82,2 & 79,9 & 85,5 & 100 & & \\
\hline 11 & 45,1 & 24,8 & 77,0 & 45,5 & 81,7 & 67,7 & 51,1 & 79,0 & 52,1 & 86,7 & 100 & \\
\hline \multirow[t]{3}{*}{12} & 63,7 & 40,1 & 77,4 & 66,5 & 81,0 & 66,4 & 67,6 & 78,3 & 64,9 & 82,2 & 77,5 & 100 \\
\hline & \multicolumn{12}{|c|}{ AFC } \\
\hline & 1 & 2 & 3 & 4 & 5 & 6 & 7 & 8 & 9 & 10 & 11 & 12 \\
\hline 1 & 100 & & & & & & & & & & & \\
\hline 2 & 16,6 & 100 & & & & & & & & & & \\
\hline 3 & 94,1 & 85,9 & 100 & & & & & & & & & \\
\hline 4 & 68,4 & 21,8 & 92,1 & 100 & & & & & & & & \\
\hline 5 & 96,5 & 99,7 & 98,4 & 96,7 & 100 & & & & & & & \\
\hline 6 & 85,3 & 85,4 & 87,8 & 83,5 & 98,4 & 100 & & & & & & \\
\hline 7 & 44,9 & 30,1 & 90,0 & 43,3 & 99,9 & 85,7 & 100 & & & & & \\
\hline 8 & 77,6 & 64,7 & 85,9 & 73,4 & 98,0 & 77,9 & 74,8 & 100 & & & & \\
\hline 9 & 38,6 & 18,4 & 93,9 & 43,4 & 98,2 & 88,8 & 55,5 & 80,0 & 100 & & & \\
\hline 10 & 85,0 & 76,9 & 84,2 & 81,7 & 98,5 & 78,9 & 82,6 & 83,7 & 89,4 & 100 & & \\
\hline 11 & 55,7 & 35,2 & 86,0 & 31,4 & 98,3 & 79,6 & 56,9 & 81,5 & 64,5 & 88,0 & 100 & \\
\hline 12 & 80,4 & 58,5 & 85,7 & 76,2 & 98,3 & 77,6 & 77,0 & 81,5 & 81,8 & 84,1 & 85,5 & 100 \\
\hline
\end{tabular}

Fuente: sobre la base de la ENEMDU de las rondas de diciembre. 


\section{REFERENCIAS}

Adepoju, A. O. (s. f.). Rural households' access to microcredit and poverty status in Obafemi- Owode local government area of Ogun State, Nigeria. Journal of Economics and Rural Development, 62-71.

Alemu, D., Bewket, W., Zeleke, G., Assefa, Y. and Trutman, P. (2011). Extent and determinants of household poverty in rural Ethiopia: A study of six villages. Eastern Africa Social Science Research Review, 21-49. doi:https://doi. org/10.1353/eas.2011.0005

Alkire, S. and Foster, J. (2007). Counting and multidimensional poverty measurement. Oxford: Oxford Poverty y Human Development Initiative (OPHI).

Alkire, S. and Foster, J. (2011). Counting and multidimensional poverty measurement. Journal of Public Economics, 95.

Alkire, S., Foster, J., Seth, S., Santos, M., Roche, J. and Ballon, P. (2015). Multidimensional poverty measurement and analysis: chapter 7-Data and Analysis. OPHI. ISBN: 978-19-0719-475-7

Anderson, F. (2018). Guía por la soberanía alimentaria. European Coordination: Vía Campesina. https://viacampesina.org/en/wp-content/uploads/ sites/2/2018/02/Food-Sovereignty-a-guide-ES-version-low-res.pdf

Apata, T. G., Apata, O. M., Igbalajobi, O. A. and Awoniyi, S. M. (2010). Determinants of rural poverty in Nigeria: Evidence from small holder farmers in South-western, Nigeria. Journal of Science and Technology Education Research, 1(4), 85-91.

Bourguignon, F., and Chakravarty, S. (2019). The measurement of multidimensional poverty. En S. Chakravarty, Poverty, Social Exclusion and Stochastic Dominance. Singapore: Springer. ISBN: 978-981-13-3431-3

Carrión, D. y Herrera, S. (2012). Ecuador rural del siglo XXI: soberanía alimentaria, inversión pública y politica agraria. Quito: Instituto de Estudios Ecuatorianos.

CEPAL. (2018). Medición de la pobreza por ingresos: actualización metodológica y resultados. Santiago: Naciones Unidas.

CEPAL/FAO/IICA. (2014). Perspectivas de la agricultura y del desarrollo rural en las Américas: una mirada hacia América Latina y el Caribe. San José. ISBN: 978-92-5-308259-9

CEPAL/ONU. (2010). Heterogeneidad estructural y brechas de productividad: de la fragmentacion a la convergencia. En La Hora de la igualdad: brechas por cerrar, caminos por abrir. Trigésimo tercer periodo de sesiones de la CEPAL (pp. 91-129). Brasilia: Naciones Unidas.

Chiriboga, M. (2015). Pequeñas economias: reflexiones sobre la agricultura familiar. Quito, Ecuador: FAO.

Chonchol, J. (2008). Globalización, pobreza y agricultura familiar. En Ruris (vol. II, pp. 184-196).

De Neubourg, C., Chai, J. and De Milliano, M. (2012). Step-by-step guidelines to the multiple overlapping deprivation analysis (MODA). Florence: UNICEF Office of Research.

FAO. (2014). El año internacional de la agricultura familiar (AIAF) 2014. Roma. http://www.fao.org/3/a-as281s.pdf

FAO. (2016). Juventud rural y empleo decente en América Latina. Santiago. Santiago. ISBN: 978-92-5-309188-1

FAO. (2018a). El trabajo de la FAO en la agricultura familiar: prepararse para el decenio internacional de agricultura familiar (2019-2028) para alcanzar los ODS.

FAO. (2018b). Erradicar la pobreza extrema: ¿Qué papel tiene la agricultura? Foro Global sobre Seguridad Alimentaria y Nutrición (p. 11). http://www.fao.org/3/ CA0989ES/ca0989es.pdf

FAO. (2018c). Panorama de la pobreza rural en América Latina y el Caribe. Santiago: 
Organización de las Naciones Unidas para la Alimentación y la Agricultura. ISBN: 978-92-5-131085-4

FAO. (s. f.). Ecuador en una mirada. FAO en Ecuador. http://www.fao.org/ecuador/ fao-en-ecuador/ecuador-en-una-mirada/es/

Flores, E. (s. f.). El modelo extractivista y los peligros para la soberanía alimentaria en el caso ecuatoriano. Universidad Nacional de General de Sarmiento.

Garner, E. y De la O Campos, P. (2014). Identifying the «family farm»: an informal discussion of the concepts and definitions. ESA Working Paper N. ${ }^{\circ}$ 14-10.

Herrera, S. (6 de julio de 2021). La agricultura familiar campesina: un modelo de agricultura con la potencia para cambiar la vida. Quito: Instituto de Estudios Ecuatorianos. https://ocaru.org.ec/2021/07/06/la-agricultura-familiar-campesina-un-modelo-de-agricultura-con-la-potencia-para-cambiar-la-vida/

Ibitola , O. R., Fasakin , I. J., Popoola, O. O. and Olajide, O. O. (2019). Poverty analysis of maize farming households in Oyo State, Nigeria. Greener Journal of Agricultural Sciences, 199-207. DOI: http://doi.org/10.15580/ GJAS.2019.2.040219063

IICA. (s. f.). Estrategia del IICA en Ecuador. http://repiica.iica.int/docs/b3676i/ b3676i.pdf

INEC. (2015a). Medición de la pobreza multidimensional en Ecuador. INEC. https:// www.ecuadorencifras.gob.ec/documentos/web-inec/Sitios/Pobreza_Multidimensional/assets/ipm-metodologia-oficial.pdf

INEC. (2015b). Reporte de pobreza por Ingresos. http://www.ecuadorencifras.gob. ec/wp-content/uploads/downloads/2015/10/Informe-pobreza-y-desigualdad-sep_15.pdf

INEC. (2015c). Compendio de resultados . Encuesta condiciones de vida ECV. Sexta ronda 2015. https://www.ecuadorencifras.gob.ec/documentos/web-inec/ECV/ ECV_2015/documentos/ECV\%20COMPENDIO\%20LIBRO.pdf

INEC. (2019). Encuesta nacional de empleo, desempleo y subempleo (ENEMDU): Indicadores laborales. https://www.ecuadorencifras.gob.ec/documentos/web-inec/ EMPLEO/2019/Marzo/032019_Mercado\%20Laboral_final.pdf

Kumar, D. (2017). Poverty and human dignity: a human rights approach. IOsR Journal of Humanities And Social Science, 48-55.

Majeed, M. T. and Malik, M. N. (2015). Determinants of household poverty: empirical evidence from Pakistan. The Pakistan Development Review, 701-717. http://www.jstor.org/stable/43831356

Maletta, H. (2011). Tendencias y perspectivas de la agricultura familiar en América Latina. Rimisp. http://www.rimisp.org/wp-content/files_mf/ 1366294106N902011AgriculturafamiliarAmericaLatinaMaletta.pdf

Martínez, L. (2013). La agricultura familiar en el Ecuador: informe del proyecto análisis de la pobreza y la desigualdad en América Latina. RIMISP. http:// rimisp.org/wp-content/files_mf/1434745799147AgriculturaFamiliarEcuadorMartinez_editado.pdf

Ministerio de Salud Pública del Ecuador. (2018). Plan intersectorial de alimentación y nutrición 2018-2025. Quito, Ecuador: Viceministerio de Gobernanza de la Salud Pública.

Okeke-Agulu, K. I., Arene, C. J. and Noble, J. N. (2014). Does livestock keeping reduce poverty among farm households in Nigeria? Journal of Agriculture and Veterinary Science (IOSR-JAVS), 31-41. e-ISSN: 2319-2380

Okidegbe, N. (2001). Rural poverty: trends and measurement. World Bank.

RIMISIP/FIDA. (2015). Situación de la educación rural en Ecuador.

Sadiq, M. S., Singh, I. P., Ahmad, M. M., Lawal, M. and Kabeer, Y. M. (2019). Cau- 
sative factors affecting livelihood status of cassava producers in Kwara State of Nigeria. Current Investigations in Agriculture and Current Research, 790-798. DOI: 10.32474/CIACR.2019.06.000233

Schejtman, A. (2008). Alcances sobre la agricultura familiar. Rimisp. http:// www.rimisp.org/wp-content/files_mf/1366379894N21_Alexander_Shejtman_doc21.pdf

Sudhakara, B. y Nega, A. (2013). Determinants of poverty in rural Tigray: Ethiopia. Evidence from rural households of Gulomekeda Wereda. International Journal of Science and Research, 822-828.

UNICEF/INEC. (2015). Trabajo infantil en Ecuador: hacia un entendimiento integral de la problématica. Offset Abad C. A.

Van Der Ploeg, J. (2014). Diez cualidades de la agricultura familiar. Revista Agriculturas: experiencias en agroecología (1), 61-65. 